\title{
A Positive Definiteness Preserving Discretization Method for nonlinear Lyapunov Differential Equations
}

\author{
Joris Gillis and Moritz Diehl
}

\begin{abstract}
Periodic Lyapunov differential equations can be used to formulate robust optimal periodic control problems for nonlinear systems. Typically, the added Lyapunov states are discretized in the same manner as the original states. This straightforward technique fails to guarantee conservation of positive-semidefiniteness of the Lyapunov matrix under discretization. This paper describes a discretization method, coined PDPLD, that does come with such a guarantee. The applicability is demonstrated at hand of a tutorial example, and is specifically suited for direct collocation methods. Keywords : optimal control, Lyapunov differential equation, robustification, collocation
\end{abstract}

\section{INTRODUCTION}

For dynamic systems that are modeled accurately by nonlinear differential equations, optimal control [1] is a useful offline technique to find open loop inputs that guide such systems along an optimal trajectory subject to path constraints. In real-world applications, modeling inaccuracy and disturbances can be treated by adding a feedback controller that tracks this trajectory. However, path constraints that are active in the offline optimal control problem solution will be impossible to satisfy with any conceivable feedback controller. Such violations can be avoided by adding safety margins to the path constraints in the optimal control problem formulation. The robust optimal control methods proposed in this paper deal with finding safety margins such that path constraints can be met during real-world operation with a certain confidence level [2], without adding unnecessary conservatism.

Typical steps in obtaining a numerical solution to an optimal control problem involve discretizing the continuous-time dynamic system, casting it as a nonlinear optimization problem, and solving it with a general nonlinear problem (NLP) solver. Robustification of the optimal control problem can be achieved by augmenting the state space of the continuous-time dynamic system with Lyapunov states [3]. Rather than treating the Lyapunov states indiscriminately from the original states - i.e. discretizing them using the same technique that served to solve the non-robustified optimal control problem up to reasonable accuracy - we propose in this paper to treat them differently. By applying discrete time Lyapunov theory on the discretized version of

J. Gillis and M. Diehl are with the Optimization in Engineering Center (OPTEC), K.U. Leuven, Kasteelpark Arenberg 10, B3001 Leuven-Heverlee, Belgium. J. Gillis is a Doctoral Fellow of the Fund for Scientific Research - Flanders (F.W.O.) in Belgium. joris.gillisdesat.kuleuven.be, moritz.diehldesat.kuleuven.be the original system, we obtain a discretization scheme for Lyapunov states that preserves positive definiteness.

Section I summarizes how the Lyapunov differential equations relate to system dynamics and state-covariance. Section II states the robust optimal control problem in continuous time and summarizes the standard method to discretize it. The main contribution of this paper is given in Section III, where an alternative discretization method with superior properties is proposed. Section IV applies the proposed method to an illustrative example. Concluding remarks are provided at the end.

\section{DYNAMIC SYSTEMS AND THE LYAPUNOV DIFFERENTIAL EQUATIONS}

Consider the class of dynamic systems that are described by an explicit time-invariant ODE:

$$
\dot{x}=f(x, u, w)
$$

with states $x \in \mathbb{R}^{n}$, control inputs $u \in \mathbb{R}^{m}$ and zero mean Gaussian disturbances $w \in \mathbb{R}^{p}$.

Throughout this paper, uncertainties are treated as Gaussian distributions. For a random variable $w$ and with $\mathrm{E}(\cdot)$ the expected value operator, we write the covariance matrix as:

$$
\Sigma_{w}=\operatorname{Cov}(w, w) \triangleq \mathrm{E}\left((w-\mathrm{E}(w))(w-\mathrm{E}(w))^{T}\right)
$$

which simplifies to $\mathrm{E}\left(w w^{T}\right)$ for zero mean distributions. The linearized covariance propagation rule through a function $g(\cdot)$ can be proven from the above definition and the knowledge that $E(\cdot)$ is a linear operator:

$$
\Sigma_{g(x)}=\frac{\partial g}{\partial x} \Sigma_{x} \frac{\partial g^{T}}{\partial x}
$$

Identifying the state-covariance matrix $\Sigma_{x(t)}$ with the Lyapunov matrix $P$, one can see that the Lyapunov differential equation describes the linearized propagation of statecovariance for continuously perturbed systems [3], [4]:

$$
\dot{P}(t)=A(t) P(t)+P(t) A^{T}(t)+B(t) \Sigma_{w} B^{T}(t)
$$

with $A=\frac{\partial f}{\partial x}$ and $B=\frac{\partial f}{\partial w}$.

The covariance matrix $\Sigma_{x} \equiv P$ defines an uncertainty ellipsoid in $n$-dimensional state space. The $s$-sigma boundary of this ellipsoid is given by the set:

$$
\left\{x_{s} \in \mathbb{R}^{n} \mid x_{s}^{T} \Sigma_{x}^{-1} x_{s}=s^{2}\right\}
$$


The eigenvectors of $\Sigma_{x}$ correspond to the principal axes of this ellipsoid and the eigenvalues are the squares of the semiaxes lengths.

The next section shows how the notion of state-covariance can be used to robustify an optimal control problem.

\section{FORMULATION AND CLASSICAL DISCRETIZATION OF ROBUST OPTIMAL CONTROL PROBLEMS}

\section{A. Formulation}

Consider the class of continuous-time $T$-periodic optimal control problems (OCP) that can be written as:

$$
\begin{array}{ccl}
\underset{x(\cdot), u(\cdot), T}{\operatorname{minimize}} & J(x(\cdot), u(\cdot), T) \\
\text { subject to } & \dot{x}(t) & =f(x(t), u(t), 0), \quad t \in[0, T] \\
x(0) & =x(T) \\
0 & \geq h_{i}(x(t)), \quad t \in[0, T] \\
& 0 & =\phi(x(0))
\end{array}
$$

where $J$ constitutes the cost functional, $h_{i}$ indicates the $i^{\text {th }}$ entry in a set of scalar path constraints of length $q$, and $\phi: \mathbb{R}^{n} \rightarrow \mathbb{R}$ is a technical requirement to remove phase invariance of the solution. In the following, for a free time in any expression, $t \in[0, T]$ shall be understood.

A solution of this open-loop OCP can be stable or unstable and can typically not be applied in real-life without further adaptations such as feedback control and safety measures. In this paper we consider a formulation of the OCP that allows both to select only stable orbits and to robustify path constraints. This formulation [3], [4] is as follows:

$$
\begin{array}{lcl}
\underset{x(\cdot), u(\cdot), P(\cdot), T}{\operatorname{minimize}} & J(x(\cdot), u(\cdot), T) \\
\text { subject to } & \dot{x}(t) & =f(x(t), u(t), 0) \\
& x(0) & =x(T) \\
\dot{P} & =A P+P A^{T}+B \Sigma_{w} B^{T} \\
P(0) & =P(T) \\
P(0) & \succeq 0 \\
0 & \geq h_{i}(x(t))+\gamma \sqrt{C_{i}(t) P C_{i}(t)^{T}} \\
& 0 &
\end{array}
$$

with $C_{i}(t)=\frac{\partial h_{i}(x(t))}{\partial x}$. We are particularly interested in solutions for which $P$ is positive-semidefinite and bounded over the period, as this corresponds to a stable trajectory [3], [4]. In this case, the Lyapunov matrix can be regarded as state-covariance and robustification is possible. Within linear approximation, the factor $\gamma$ ensures that the path-constraints at any given time are satisfied to a tolerance of $\gamma$-sigma.

The classical way to solve Problem (6) is by augmenting the state space [3]. One picks a set of Lyapunov states $y \in \mathbb{R}^{l}$ that parametrize the Lyapunov matrix $P \in \mathbb{R}^{n \times n}$ by means of a mapping $L: \mathbb{R}^{l} \rightarrow \mathbb{R}^{n \times n}$ and adds these states to the original state-space.

The remainder of this section uses the symbolics of Problem (5), and applies as such equally well to non-robustified optimal control problems as to robustified problems cast by the above transformation.

\section{B. Direct transcription}

In the family of direct methods, one proceeds by discretizing the infinite-dimensional problem such that it becomes a finite-dimensional nonlinear problem. Consider a global time-grid $\left[t_{0}, t_{1}, \ldots, t_{N}\right]^{T}$ of monotonously increasing time instants. The states sampled at these instants are denoted by $x_{0}, x_{1}, \ldots x_{N}$, sampled control inputs as $u_{0}, u_{1}, \ldots u_{N-1}$ and sampled disturbances as $w_{0}, w_{1}, \ldots w_{N-1}$.

For any type of integrator, the action of integrating the system $f$ can be formulated as a discrete time system:

$$
x_{k+1}=\Phi\left(t_{k+1}-t_{k} ; x_{k}, u_{k}, w_{k}\right), \quad k=0 \ldots N-1
$$

With this abstraction and assuming an evenly spaced time discretization, Problem (5) can be discretized as:

$$
\begin{aligned}
& \min _{x_{\bullet}, u_{\bullet}, \delta} J\left(x_{\bullet}, u_{\bullet}, \delta N\right) \\
& \text { s.t. } \quad x_{k+1}=\Phi\left(\delta ; x_{k}, u_{k}, 0\right), \quad k=0 \ldots N-1 \\
& x_{0}=x_{N} \\
& 0=\phi\left(x_{0}\right) \\
& 0 \geq h_{i}\left(x_{k}\right), \quad k=0 \ldots N-1
\end{aligned}
$$

with $\delta=\frac{T}{N}$.

If the function $\Phi$ is obtained by an underlying integrator, this formulation amounts to what is called a direct multipleshooting method [5]. This numerical scheme was applied to solve the OCP with Lyapunov states in [4], [3].

\section{Collocation integrator}

A collocation integrator is a particular numerical scheme to perform the integrating action $\Phi$. For each control interval $k$, a collocation scheme of degree $d$ introduces helper state samples $x_{k, 0}, x_{k, 1}, \ldots x_{k, d}$ sampled on times $\delta\left(k+\rho_{0}\right), \delta(k+$ $\left.\rho_{1}\right), \ldots \delta\left(k+\rho_{d}\right)$ with $\rho \in[0,1]$ a local timebase on the control interval. For convenience, we group these helper state samples into a single vector:

$$
z_{k}=\left(\begin{array}{c}
x_{k, 0} \\
\cdots \\
x_{k, d}
\end{array}\right) \in \mathbb{R}^{n(d+1)}
$$

Collocation deals with a polynomial $\Pi\left(\bullet ; z_{k}\right): \mathbb{R} \rightarrow \mathbb{R}^{n}, \rho \mapsto$ $\Pi\left(\rho ; z_{k}\right)$ that interpolates between these samples. The interpolation is exact:

$$
x_{k, j} \equiv \Pi\left(\rho_{j} ; z_{k}\right), \quad j=0 \ldots d, \quad k=0 \ldots N-1
$$

To perform integration with this polynomial, we require firstly that the polynomial evaluated at the start of the control interval should match the initial value for that interval, and secondly that derivatives of the interpolating polynomial should match the state dynamics at each point of the local timebase:

$$
G=\left(\begin{array}{c}
\Pi\left(0 ; z_{k}\right)-x_{k} \\
\frac{\partial \Pi}{\partial \rho}\left(\rho_{1} ; z_{k}\right)-\delta f\left(\Pi\left(\rho_{1} ; z_{k}\right), u_{k}, w_{k}\right) \\
\ldots \\
\frac{\partial \Pi}{\partial \rho}\left(\rho_{d} ; z_{k}\right)-\delta f\left(\Pi\left(\rho_{d} ; z_{k}\right), u_{k}, w_{k}\right)
\end{array}\right)=0
$$


This set of $n(d+1)$ nonlinear equations implicitly defines a solution for the helper sample states: $z_{k}^{*}=G^{-1}\left(\delta ; x_{k}, u_{k}, w_{k}\right)$. Lastly, the final value on the control interval $k$ can be obtained as $\Pi\left(1 ; z_{k}^{*}\right)$.

As such, collocation is a specific implicit integration scheme that reads:

$$
\begin{cases}x_{k+1} & =F\left(z_{k}\right) \\ 0 & =G\left(\delta ; x_{k}, z_{k}, u_{k}, w_{k}\right)\end{cases}
$$

Where the function $F: \mathbb{R}^{n \times(d+1)} \rightarrow \mathbb{R}^{n}$ is simply:

$$
F=\Pi\left(1 ; z_{k}\right)
$$

It is an important observation that Equation (12) is equivalent to a nonlinear discrete time system $x_{k+1}=\Phi_{\text {coll }}\left(\delta ; x_{k}, u_{k}, w_{k}\right)$.

\section{Direct collocation method}

If we replace the explicit function $\Phi$ in Equation (8) with the implicit form $(F, G)$, we effectively pass on the burden of solving the nonlinear implicit functions at each control interval onto the global nonlinear problem arising from the multiple shooting approach, yielding a direct collocation method:

$$
\begin{array}{lcl}
\min _{x_{\bullet}, u_{\bullet}, z_{\bullet}, \delta} & J\left(x_{\bullet}, u_{\bullet}, \delta N\right) \\
\text { s.t. } & x_{k+1}=F\left(z_{k}\right) \\
& 0 & =G\left(\delta ; x_{k}, z_{k}, u_{k}, 0\right) \\
& x_{0} & =x_{N} \\
& 0 & =\phi\left(x_{0}\right) \\
& 0 & \geq h_{i}\left(x_{k}\right), \quad k=0 \ldots N-1 .
\end{array}
$$

While this method is applied successfully to non-robustified optimal control problems for a wide range of practical engineering problems [6], the following section argues that its application to robust optimal control problems cast into nonrobust form is problematic. We instead propose an alternative method.

\section{POSITIVE DEFINITENESS PRESERVING LYAPUNOV DISCRETIZATION}

Applying the collocation scheme to the augmented state space formulation is straightforward, but comes with a drawback: there is no guarantee that a positivesemidefinite $P$ at any one point in the trajectory results in $P$ being positive-semidefinite in the entire trajectory. The continuous Lyapunov equation (3) provides such a guarantee in continuous time, but this guarantee is lost during discretization due to limited integration accuracy. It can indeed be observed that a discretization sufficiently accurate for a non-robustified collocation optimal control problem can easily be too coarse for a corresponding robustified problem to keep $P$ positive-semidefinite in practice, leading the nonlinear solver to fail to find a feasible solution. The technique proposed here, Positive Definiteness Preserving Lyapunov Discretization (PDPLD), tackles this problem.
We take a step back and reason about the propagation of covariance directly onto discretized Problem (8) in the original state space. In a linear approximation over one control interval, the state covariance matrix evolves as:

$$
P_{k+1}=\frac{\partial \Phi}{\partial x} P_{k} \frac{\partial \Phi^{T}}{\partial x}+\frac{\partial \Phi}{\partial w} \Sigma_{w} \frac{\partial \Phi^{T}}{\partial w}
$$

Applying the implicit function theorem to Equation (12) that defines $\Phi_{\text {coll }}$ in an implicit manner, leads to the following identities:

$$
\begin{gathered}
\frac{\partial \Phi_{\text {coll }}}{\partial x}=\underbrace{-\frac{\partial F}{\partial z}\left(\frac{\partial G}{\partial z}\right)^{-1}}_{\triangleq M} \frac{\partial G}{\partial x} \\
\frac{\partial \Phi_{\text {coll }}}{\partial w}=M \frac{\partial G}{\partial w}
\end{gathered}
$$

Avoiding symbolic inversion, we propose to introduce a helper variable $M \in \mathbb{R}^{n \times n(d+1)}$ and obtain an implicit integrator scheme for both original and Lyapunov states, which we will refer to as PDPLD:

$$
\left\{\begin{aligned}
x_{k+1} & =F\left(z_{k}\right) \\
0 & =G\left(\delta ; x_{k}, z_{k}, u_{k}, w_{k}\right) \\
P_{k+1} & =M_{k}\left(\frac{\partial G}{\partial x} P_{k} \frac{\partial G^{T}}{\partial x}+\frac{\partial G}{\partial w} \Sigma_{w} \frac{\partial G^{T}}{\partial w}\right) M_{k}^{T} \\
0 & =\frac{\partial F^{T}}{\partial z}-\frac{\partial G^{T}}{\partial z} M_{k}^{T}
\end{aligned}\right.
$$

The lemma that accompanies this scheme reads:

Lemma 1: If $P_{0} \succeq 0$ and $P_{0} \ldots P_{N}$ satisfy Equation (18), then $P_{1} \ldots P_{N} \succeq 0$.

Assuming $\Sigma_{w} \succeq 0$ and starting from $P_{0} \succeq 0$, the lemma follows by induction using Equation (18c)

Starting from a non-robustified optimal-control problem discretized using direct collocation as in Formulation (14), we propose now to robustify with PDPLD such that Formulation (19) is obtained with the following decision variables:

$$
\begin{array}{ll}
x_{k} \in \mathbb{R}^{n}, P_{k} \in \mathbb{R}^{n \times n}, & k=0 \ldots N \\
u_{k} \in \mathbb{R}^{m}, z_{k} \in \mathbb{R}^{n(d+1)}, M_{k} \in \mathbb{R}^{n \times n(d+1)}, & k=0 \ldots N-1 \\
T \in \mathbb{R} &
\end{array}
$$

The lemma that accompanies this formulation reads:

Lemma 2: If Formulation (19) has a feasible solution, and if a technical assumption is satisfied, then the nonlinear discrete time system (12) is asympotically stable along the periodic open loop orbit.

Technical Assumption: The system is controllable along the orbit with respect to the noise in the sense that the discrete time Lyapunov equation, if started with $P_{0}=0$, would result in a strictly positive definite $P_{N}$.

Proof: Given the fact that the discrete time Lyapunov equation is exact for the discrete time system and admits 


\begin{tabular}{|c|c|c|c|c|c|}
\hline$\underset{x_{\bullet}, u_{\bullet}, z_{\bullet}, P_{\bullet}, M_{\bullet}, T}{\operatorname{minimize}}$ & $J\left(x_{\bullet}, u_{\bullet}, T\right)$ & & & & \# \\
\hline \multirow[t]{9}{*}{ subject to } & $x_{k+1}$ & $=$ & $F\left(z_{k}\right)$ & & $n N$ \\
\hline & 0 & $=$ & $G\left(T / N ; x_{k}, z_{k}, u_{k}, 0\right)$ & & $N n(d+1)$ \\
\hline & $x_{0}$ & $=$ & $x_{N}$ & & $n$ \\
\hline & $P_{k+1}$ & $=$ & $M_{k}\left(\frac{\partial G}{\partial x} P_{k} \frac{\partial G}{\partial x}^{T}+\frac{\partial G}{\partial w} \Sigma_{w} \frac{\partial G}{\partial w}^{T}\right) M_{k}^{T}$ & & $n^{2} N$ \\
\hline & $P_{0}$ & $=$ & $P_{N}$ & & $n^{2}$ \\
\hline & & $\succeq$ & 0 & & $n$ \\
\hline & $\frac{\partial F}{\partial z}^{T}\left(z_{k}\right)$ & $=$ & $\frac{\partial G}{\partial z}^{T}\left(x_{k}, z_{k}, u_{k}, 0\right) M_{k}^{T}$ & & $N n^{2}(d+1)$ \\
\hline & 0 & $=$ & $\phi\left(x_{0}\right)$ & & 1 \\
\hline & 0 & $\geq$ & 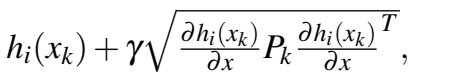 & $\begin{array}{l}i=0 \ldots q-1 \\
k=0 \ldots N-1\end{array}$ & $N q$ \\
\hline
\end{tabular}

a feasible periodic solution which is positive semidefinite, the Lyapunov Lemma [4] together with the technical assumption implies that the linearized discrete time system is asymptotically stable. Asymptotic stability of the nonlinear system in the neighborhood of the periodic orbit follows.

The benefit of the PDPLD integration scheme is illustrated below. Consider an autonomous nonlinear oscillator,

$$
\frac{\mathrm{d}}{\mathrm{d} t}\left(\begin{array}{l}
Y \\
Z
\end{array}\right)=\left[\begin{array}{cc}
0 & 1 \\
-\kappa & -c
\end{array}\right]\left(\begin{array}{l}
Y \\
Z
\end{array}\right)+\left(\begin{array}{c}
0 \\
-\alpha Z^{2}
\end{array}\right) .
$$

For $\alpha=0$, the under-dampened system exhibits a natural frequency of

$$
\omega=\sqrt{\kappa-\frac{c^{2}}{4}} .
$$

We compare classical collocation (integration scheme (12) applied to augmented state space (6)) and PDPLD collocation (integration scheme (18)) in Figure 1 with a reference solution obtained from high accuracy $\left(1 \times 10^{-12}\right)$ variable step-size integration with SUNDIALS [7].

For classical collocation, the zero-crossing of the plotted minimal eigenvalue of $P$ demonstrates a disruptive change in definiteness. In contrast, PDPLD collocation features a Lyapunov matrix that remains positive semi-definite and remains very close to the reference solution.

\section{APPLICATION IN ROBUST OPTIMAL CONTROL}

The remainder of this paper shows the application of Formulation (19) to a simple robust optimal control problem that was specifically constructed to allow for intuitive interpretation of results.

The robust optimal control problem is solved by a homotopy in three consecutive steps. Each step reuses the primal and dual solution of the previous step where possible. An SQP method is ideally suited to execute such a homotopy.

Step 1: A nominal trajectory is identified by solving Problem (14) for the nominal system.

Step 2: $P$ and $M$ are introduced as in Problem (19) but with non-robustified path constraints. This avoids

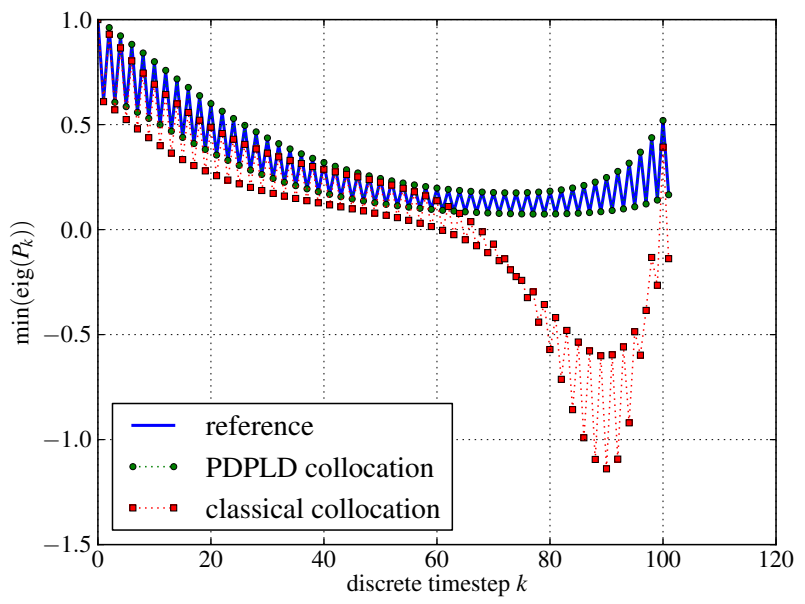

Fig. 1: Comparison of integration schemes for the nonlinear oscillator system with $\kappa=10, c=-0.02, \alpha=-0.1$, discretization time $T=\frac{\pi}{\omega}$ and initial state $z(0)=1, z^{\prime}(0)=0$. A Legendre polynomial of degree $d=5$ is chosen [8].

domain errors in the path constraints resulting from nonpositive-definiteness of $P$ in the intermediate iterations.

Step 3: The full Problem (19) is tackled.

\section{A. Implementation details}

The problem is formulated in the Python scripting environment. Gradients and Hessians are constructed by efficient sparsity-aware automatic differentiation provided by CasADi [9]. The nonlinear SQP solver WORHP [10] is used to solve the resulting nonlinear program with a tolerance of $1 \times 10^{-10}$. The linear solver used with WORHP is MA57 [11].

\section{B. Problem statement}

The problem at hand consists of a point mass moving in a plane. The plane contains obstacles (super-ellipses) that the point may not intrude. The goal is to find a time-optimal periodic trajectory for the point mass in the plane, initialized with trivial initial guess. The control inputs are coordinates of a guide-point. The point mass is connected with a spring to the guide-point. The point mass is subject to friction 
that stabilizes the system. The disturbances are forces on the point mass. This system behaves like a 3D pendulum, suspended from the guide-point.

We define the following system components:

- States: $x=\left[\begin{array}{llll}p_{x} & p_{y} & v_{x} & v_{y}\end{array}\right]^{T}, \quad n=4$

- Controls: $u=\left[\begin{array}{ll}u_{x} & u_{y}\end{array}\right]^{T}, \quad m=2$

- Disturbances: $w=\left[\begin{array}{ll}w_{x} & w_{y}\end{array}\right]^{T}, \quad p=2$

The system dynamics $\dot{x}=f(x, u, w)$ is described by:

$$
\dot{x}=\left(\begin{array}{c}
v_{x} \\
v_{y} \\
-\kappa\left(p_{x}-u_{x}\right)-\beta v_{x} \sqrt{v_{x}^{2}+v_{y}^{2}+c^{2}}+w_{x} \\
-\kappa\left(p_{y}-u_{y}\right)-\beta v_{y} \sqrt{v_{x}^{2}+v_{y}^{2}+c^{2}}+w_{y}
\end{array}\right)
$$

As parameters we use $\kappa=10, c=1$ and $\beta=1$.

The path constraints are given by:

$$
h_{i}\left(p_{x, k}\right)=\left(\frac{p_{x, k}-x_{0, i}}{a_{i}}\right)^{n_{i}}+\left(\frac{p_{y, k}-y_{0, i}}{b_{i}}\right)^{n_{i}}-1
$$

with $n_{i} \in \mathbb{N}^{+}$. We use the following numerical values:

\begin{tabular}{l|lllll}
$\mathrm{i}$ & $x_{0, i}$ & $y_{0, i}$ & $a_{i}$ & $b_{i}$ & $n_{i}$ \\
\hline 0 & 0 & 0 & 1 & 1 & 4 \\
1 & 1 & 0.5 & 0.5 & 2 & 4
\end{tabular}

The objective function is chosen to be time-optimality, with added regularization of control inputs:

$$
J\left(x_{\bullet}, u_{\bullet}, T\right)=T+\frac{1 \times 10^{-2}}{2 N} \sum_{k=0}^{N-1}\left\|u_{k}\right\|_{2}^{2}
$$

The phase fix function is chosen to be:

$$
\phi(x)=p_{x}
$$

The number of control intervals is chosen as $N=40$. A Legendre polynomial of degree $d=5$ is chosen [8].

\section{Homotopy results}

Problem (14) is instantiated with the above particularities. All decision variables are initialized by zero, with two exceptions: $T=4$, and the parts of the states $x$ and helper states $z$ that correspond to coordinates in the plane are initialized by a circle of radius 3 , encircling the obstacles by a wide margin.

The initialization is illustrated in Figure 2. Some iterates are shown in Figure 3 while the converged solution is shown in Figure 4. Active path constraints are highlighted by a line originating from the obstacle center.

In the next step, $P$ and $M$ are introduced as in Problem (19) with non-robustified path constraints. The disturbance is chosen to have covariance $\Sigma_{w}=I$. Figure 5 shows the solution to Step 2.

A last step adds the robustifying terms to the path constraints with $\gamma=1$. Figure 6 shows the solution to Step 3 .

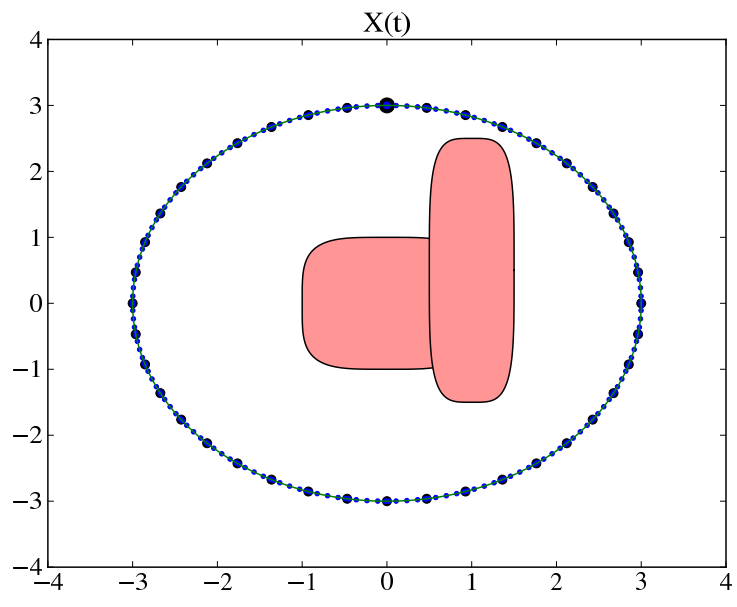

Fig. 2: Illustration of the initial guess of Step 1. This plot shows the plane with the two obstacles. One can discern 40 black circles that correspond to $x_{\bullet}$ and tiny dots in between that correspond to $z_{\bullet}$. The polynomial interpolation of the collocation can be seen in the background. The highlighted dot at the top corresponds to $k=0$ where the phase fix is active. The motion is clock-wise around the obstacles.

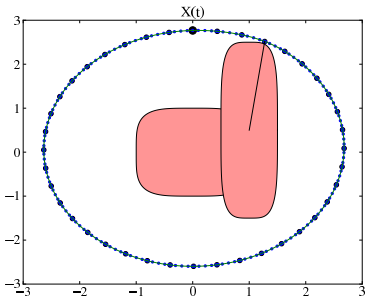

(a) Iteration 2 .

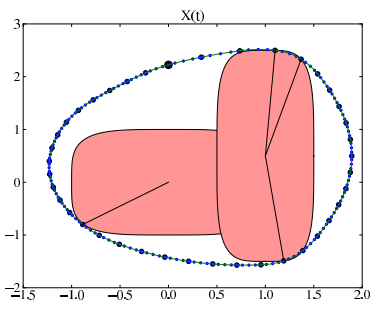

(c) Iteration 19.

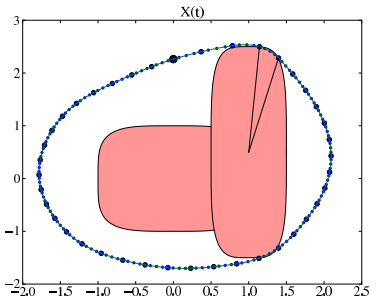

(b) Iteration 14 .

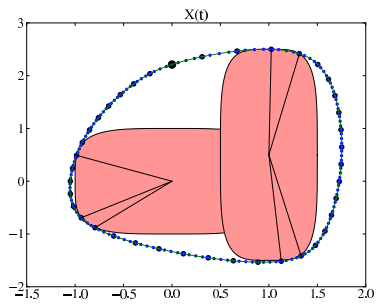

(d) Iteration 39.
Fig. 3: Intermediate trajectories during WORHP iterations of Step 1.

Some remarks are in place when closely observing the solution of this application in Figure 7.

First, it is apparent that the interpolated trajectory from Step 1 intersects with the obstacle. Indeed, during discretization, the path constraints were only sampled at a few selected locations. There is no guarantee of non-intersection for other points on this curve.

Second, at the locations where the robustified path constraints are active, the uncertainty ellipsoids are not touching the 

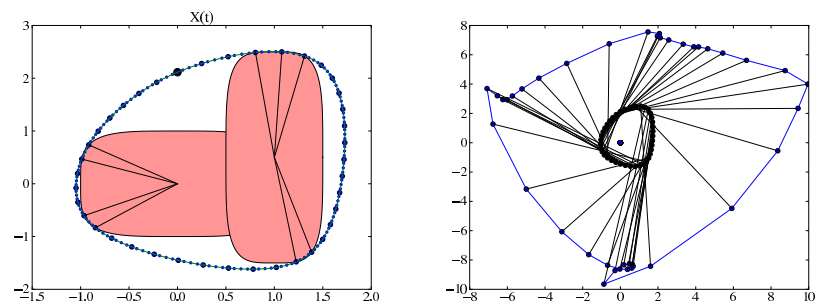

Fig. 4: Fully converged Step 1. The right figure shows control inputs. The springs that connect the guide-point with the point mass are drawn for each control interval.

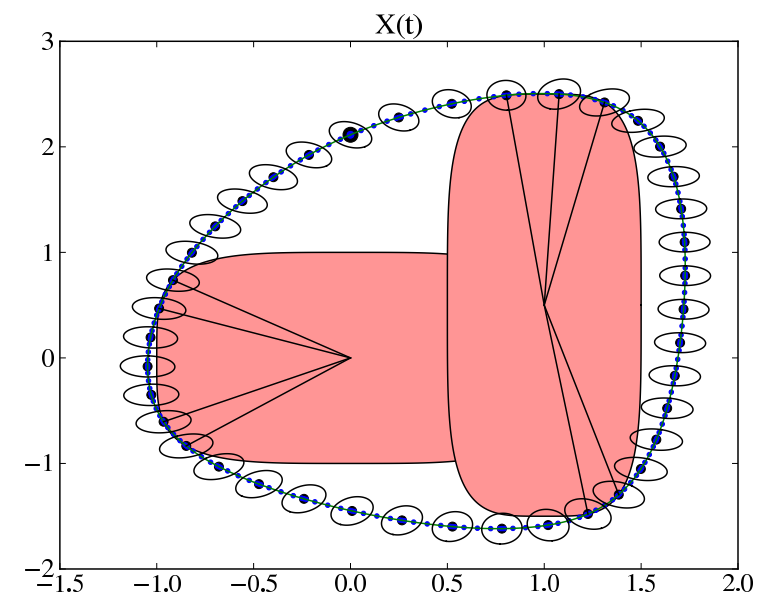

Fig. 5: Illustration of converged Step 2: covariances propagated without robustification. The 1-sigma bounds of the uncertainty ellipsoids are highlighted.

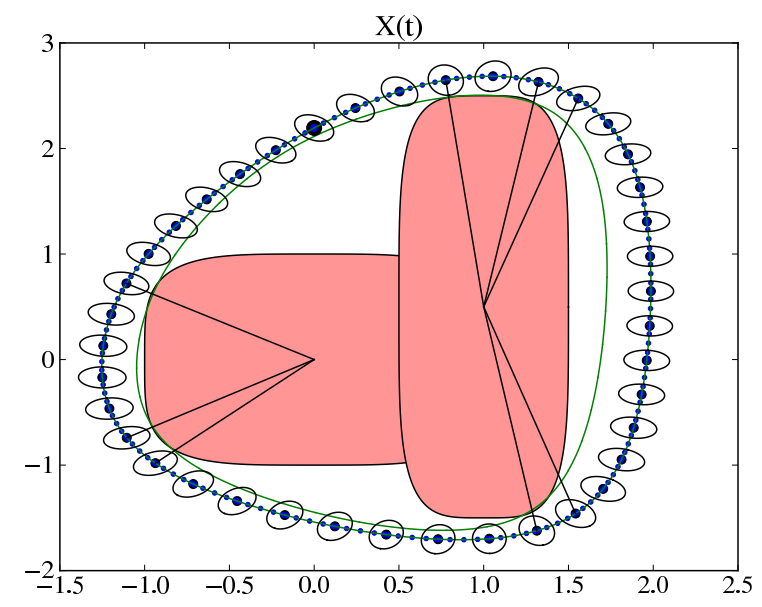

Fig. 6: Illustration of converged Step 3. The interpolated path of non-robustified Step 1 is shown in the background.

obstacle. Indeed, a min-max formulation is required to obtain such geometrically sound result. It cannot be expected from the linearization based robustifying formulation used here. The robustifying term of Equation (6) only accounts for uncertainty in the $\frac{\partial h_{i}}{\partial x}$ direction and is subject to linearization errors of the nonlinear constraint function.

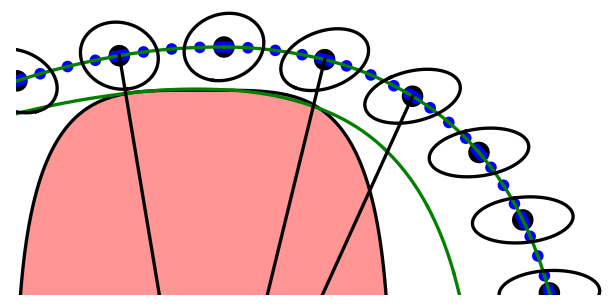

Fig. 7: Focusing on the upper region of Figure 6.

\section{CONCLUSION}

In this paper, a Positive Definiteness Preserving Lyapunov Discretization (PDPLD) was proposed to treat Lyapunov differential equations. It allows to use direct collocation to solve robust OCPs. A three step practical procedure was suggested that led to a successful application of the proposed method to a tutorial example.

\section{ACKNOWLEDGMENTS}

This research was supported by Research Council KUL: PFV/10/002 Optimization in Engineering Center OPTEC, GOA/10/09 MaNet and GOA/10/11 Global real- time optimal control of autonomous robots and mechatronic systems. Flemish Government: IOF / KP / SCORES4CHEM, FWO: PhD/postdoc grants and projects: G.0320.08 (convex MPC), G.0377.09 (Mechatronics MPC); IWT: PhD Grants, projects: SBO LeCoPro; Belgian Federal Science Policy Office: IUAP P7 (DYSCO, Dynamical systems, control and optimization, 2012-2017); EU: FP7- EMBOCON (ICT-248940), FP7-SADCO ( MC ITN-264735), ERC ST HIGHWIND (259 166), Eurostars SMART, ACCM.

\section{REFERENCES}

[1] M. Athans and P. L. Falb, Optimal Control - An Introduction to the Theory and Its Applications. Dover, 1966.

[2] K. Zhou, J. Doyle, and K. Glover, Robust and optimal control. Englewood Cliffs, NJ: Prentice Hall, 1996.

[3] B. Houska and M. Diehl, "Robustness and Stability Optimization of Power Generating Kite Systems in a Periodic Pumping Mode," in Proceedings of the IEEE Multi - Conference on Systems and Control, (Yokohama, Japan), pp. 2172-2177, 2010.

[4] P. Bolzern and P. Colaneri, "The periodic Lyapunov equation," SIAM J. Matrix Anal. Appl., vol. 9(4), pp. 499-512, 1988.

[5] H. Bock and K. Plitt, "A multiple shooting algorithm for direct solution of optimal control problems," in Proceedings 9th IFAC World Congress Budapest, pp. 243-247, Pergamon Press, 1984.

[6] F. Magnusson, "Collocation Methods in JModelica.org," Master's thesis, Department of Automatic Control, Lund University, Sweden, 2012.

[7] A. Hindmarsh, P. Brown, K. Grant, S. Lee, R. Serban, D. Shumaker and C. Woodward, "SUNDIALS: Suite of nonlinear and differential/algebraic equation solvers," ACM Transactions on Mathematical Software, vol. 31, pp. 363-396, 2005.

[8] L. T. Biegler, Nonlinear Programming. MOS-SIAM Series on Optimization, SIAM, 2010.

[9] J. Andersson, J. Åkesson, and M. Diehl, "CasADi - A symbolic package for automatic differentiation and optimal control," in Recent Advances in Algorithmic Differentiation (S. Forth, P. Hovland, E. Phipps, J. Utke, and A. Walther, eds.), Lecture Notes in Computational Science and Engineering, (Berlin), Springer, 2012.

[10] C. Büskens and D. Wassel, Modeling and Optimization in Space Engineering, ch. The ESA NLP Solver WORHP. Springer Verlag, 2012.

[11] "Hsl(2011). a collection of fortran codes for large scale scientific computation. http://www.hsl.rl.ac.uk," 2011. 